\title{
Comparison of Ultrasonogram (USG)- Guided Supraclavicular Approach vs Infraclavicular Approach for Subclavian Vein (SCV) Cannulation
}

\author{
Authors \\ Saravanakumar $\mathbf{S}^{1}$, Satish Logidasan ${ }^{2}$, Kanimozhi Rathinasamy ${ }^{3}$ \\ Department of Anesthesiology, Govt Stanley Medical College, TN- 600001, India \\ Corresponding Author \\ Kanimozhi Rathinasamy \\ Department of Anesthesiology, Govt Stanley Medical College, TN- 600001, India
}

\begin{abstract}
Background: Central venous cannulation is essential for open heart surgery and critically ill patients. SCV was customarily cannulated by infraclavicular landmark technique. With USG, waves are obscured by bony structures in infraclavicular but clear with supraclavicular approach. Therefore we compared these approaches.

Materials and Methods: 40 patients undergoing open heart surgery were randomly allocated into Group $S$ and Group I. Group S(n=20) underwent SCV cannulation by supraclavicular approach, GroupI $(n=20)$ by infraclavicular approach. Outcome measures are duration of the procedure, number of attempts, success of cannulation and the complications. Under USG guidance, in supraclavicular approach the vein was cannulated by 'in plane technique' and longitudinal axis, in infraclavicular approach by 'out of plane' technique and short axis of the vein.

Results: The duration of the procedure (secs) for supraclavicular and infraclavicular approaches were $68.53+28.39$ and $116.58+38.95$ respectively with $P$ value of $<0.05$, hence statistically significant. The number of successful attempts for supraclavicular and infraclavicular approaches were $1.26+0.375$ and $1.21+0.42$ respectively and the success rate was $95.0 \%$ for both approaches.

Conclusion: USG guided SCVcannulation is easy and rapid to perform with supraclavicular approach.

Keywords: SCV cannulation, Supra clavicular, Infraclavicular, Ultrasound guidance.
\end{abstract}

\section{Introduction}

Central venous cannulation is essential for open heart surgery and critically ill patients. USG is used for SCV and IJV cannulation. SCV can be cannulated by supraclavicular and infraclavicular approaches. SCV was customarily cannulated by infraclavicular landmark technique. With USG guidance sound waves are obscured by bony structures in infraclavicular but clear with supraclavicular approach. Therefore we compared these two approaches.

\section{Materials and Methods}

40 patients undergoing open heart surgery were randomly allocated into Group S and Group I. Group S $(n=20)$ underwent SCV cannulation by supraclavicular approach, Group I $(n=20)$ by infraclavicular approach. Outcome measures 
areduration of the procedure, number of attempts, success of cannulation and the complications. Under USG guidance, in supraclavicular approach the vein was cannulated by 'inplane technique' and longitudinal axis, in infraclavicular approach by 'out of plane' technique and short axis of the vein.

Fig 1. Duration of the procedure

\begin{tabular}{|l|c|}
\hline Approach & $\begin{array}{c}\text { Average Duration of the } \\
\text { Procedure (secs) }\end{array}$ \\
\hline Supraclavicular & 68.53 \\
\hline Infraclavicular & 116.58 \\
\hline
\end{tabular}

Fig 2. Attempts for the procedure

\begin{tabular}{|l|c|c|c|}
\hline \multirow{2}{*}{ Approach } & \multicolumn{2}{|c|}{ Arterial Puncture } & \multirow{2}{*}{ Total } \\
\hline Supraclavicular & Yes & No & \\
\hline Infraclavicular & $1(5 \%)$ & $18(90 \%)$ & 20 \\
\hline Total & 3 & 37 & 40 \\
\hline
\end{tabular}

Fig 3. Success Rates after both approaches

\begin{tabular}{|l|c|c|c|}
\hline Approach & Success & Failed & Total \\
\hline Infraclavicular & $19(95 \%)$ & $1(5 \%)$ & 20 \\
\hline Supraclavicular & $19(95 \%)$ & $1(5 \%)$ & 20 \\
\hline Total & 38 & 2 & 40 \\
\hline
\end{tabular}

Fig 4. Rate of arterial puncture

\begin{tabular}{|l|c|c|c|c|}
\hline \multirow{2}{*}{ Approach } & \multicolumn{3}{|c|}{ No. of Attempts } & \multirow{2}{*}{ Total } \\
\cline { 2 - 4 } & 1 & 2 & $>2$ & \\
\hline Infraclavicular & 15 & 4 & 1 & 20 \\
\hline Supraclavicular & 16 & 3 & 1 & 20 \\
\hline Total & 31 & 7 & 2 & 40 \\
\hline
\end{tabular}

\section{Results}

Statistical analysis was done by applying Chi-square test and Student's Independent Samples T-test.

The number of successful attempts for supraclavicular and infraclavicular approaches were $1.26+$ 0.375 and $1.21+0.42$ respectively $(\mathrm{P}>0.05)$.

The duration of the procedure (secs) for supraclavicular and infraclavicular approaches were $68.53+28.39$ and $116.58+38.95$ respectively with $\mathrm{p}$ value $<0.05$, difference was statistically significant.
The overall success rate was $95.0 \%$ for supraclavicular and $95.0 \%$ for infraclavicular approach $(\mathrm{p}>0.05)$.

\section{Discussion}

The results reported that the duration of procedure was relatively higher in infraclavicular approach. Our observation of longer duration in infraclavicular approach may be due to difficult sonoanatomy caused by bony shadows. However with this approach arterial puncture is minimized due to the simultaneous visualization of artery and vein.

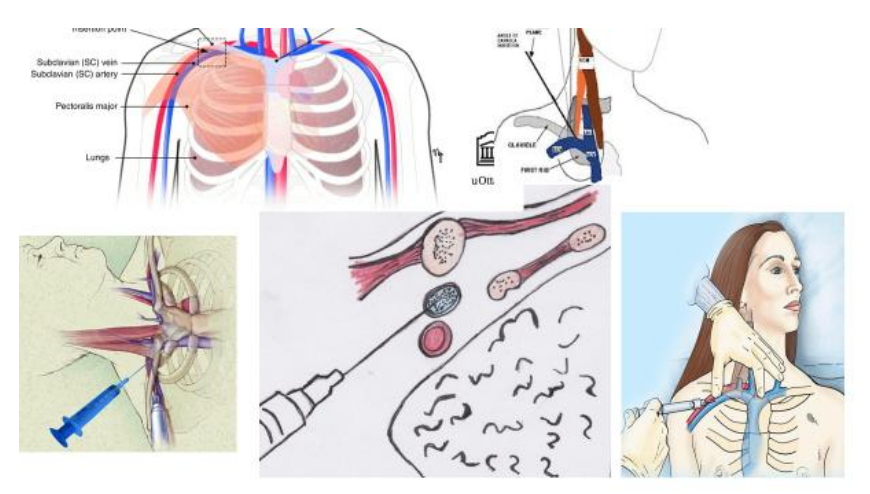

Fig 5. Inplane and out of plane Subclavian vein canulation.

Both the approaches has merits and demerits in them. The advantages in supraclavicular approach is that the vein is at a short distance from the skin and the needle in inplane orientation to the probe, visualizing the whereabouts of needle tip whereas in infraclavicular approach artery and vein both are visualized simultaneously. The disavantages in supraclavicular approach is that the artery is not visualized simultaneously and its relationship with lung while in infraclavicular approach the needle is in out of plane orientation leading to difficulty in needle tip visualization and the depth of the vein from skin.

Vascular delineation can be enhanced by colour Doppler imaging. Needle movements can be well followed with newer needle navigation systems.

\section{Conclusion}

The duration of subclavian vein cannulation by supraclavicular approach is significantly shorter 
than the infraclavicular approach. However this statement need to be confirmed by meta analytic studies.

\section{References}

1. A novel technique for ultrasound-guided supraclavicular subclavian cannulation. Am J Emerg Med. 2010 Oct;28(8):966-9. doi: 10.1016/j.ajem.2009.07.019. Epub 2010 Jan28.

2. Real-time ultrasound-guided subclavian vein cannulation versus the landmark method in critical care patients: a prospective randomized study. Crit Care Med. 2011 Jul;39(7):1607- 12 . doi: 10.1097/CCM.0b013e318218a1ae.

3. Ultrasound-guided infraclavicular axillary vein cannulation for central venous access. British Journal of Anaesthesia 93 (2): 18892 DOI: 10.1093/bja/aeh187 Advance Access publication June 25, 20041.

4. Asheim P, Mastad U, Aadahl P. Ultrasoundguided central venouscannulation in infants and children. Acta Anaesthesiol Scand 2002; 46: $390-2$

5. O'Grady NP, Alexander M, Dellinger EP, et al: Guidelines for prevention of intravascular catheter-related infections. MMWR Morb Mortal Wkly Rep 51:1-29, 2002.

6. Consecutive, prospective case series of a new method for ultrasound-guided supraclavicular approach to the brachiocephalic vein in children British Journal of Anaesthesia 106 (5): 732-7 (2011) Advance Access publication $17 \quad 2011$ doi:10.1093/bja/aer031 